\title{
Postpartum depression: Risks and early detection
}

Postpartum depression, also known as postnatal depression, is a common condition among mothers in the first month following childbirth, or sometimes even for a longer period. According to global data, its frequency ranges from $10 \%$ to $20 \%$, although it may be higher and reach $26 \%$, as observed in teenage and single mothers and, especially, even more in those who have a low social status, a low level of education or who are poor or malnourished. This situation reflects that one in every four mothers have postpartum depression, although it may not be severe.

The Diagnostic and Statistical Manual of Mental Disorders, fifth edition (DSM-V), indicates that a major depressive psychiatric disorder occurs when symptoms are observed already during pregnancy, whereas women with a lower risk usually develop symptoms in the first weeks following delivery.

The consequences of such depressive disorder especially affect the mother's well-being, although it may also result in little care provided to the baby since the first days of life, in particular if the mother is unable to breastfeed them or refuses to do it. It is also common that these mothers are incapable of approaching and speaking to their baby, which is what mothers usually do. Sometimes, their disorder may also have an impact on the baby's father and other family members, although this is uncommon.

In addition, risk groups have been identified among mothers, especially those who have disorders during pregnancy and are observed to have a greater vulnerability. However, the most common postpartum depression occurs in the first month, although it may extend up to the first three months after childbirth. During such periods, it is critical for physicians to help the mothers, before and after delivery, especially in the case of obstetricians and pediatricians, whom the mothers know well and trust for help. To this end, during an office visit, physicians should implement a sensitive approach and listen to the mothers as much as possible; this way, they will be able to detect any mental health symptom. If this occurs during postpartum office visits, either with the obstetrician or pediatrician, they will need to perform a comprehensive assessment so that they are able to progressively find the two more common symptoms: depression with a low mood and lack of interest in their baby.
The causes of postpartum depression are not very clear, but the following factors may be useful to detect who is at a higher risk. The most frequent disorders observed include depression during a previous pregnancy or postnatal period, onset of symptoms during current pregnancy, deep sadness, anxiety, frequent crying, mood changes, irritability, feeling overwhelmed, sleep alteration, stress due to the absence of the father during pregnancy, remaining alone after childbirth, and lack of family support.

Symptoms may be similar to those of a major depression and usually also include worries and fears about being a mother. In this situation, it is necessarily appropriate to tell the mothers that they should receive psychological counseling, which may help them to improve in the term of three to four weeks. In addition, if depression persists after the first few months, psychiatrists tend to prescribe antidepressants, which may be beneficial, and improvements can be observed approximately at 30 days. An even more severe but very uncommon condition may also occur, known as postpartum psychosis or puerperal psychosis, which requires psychiatric management as soon as detected. In addition, several studies have pointed out that the higher risk takes place when postpartum depression occurs in mothers with several problems but mainly when depression was already present during pregnancy and was left untreated.

It has also been observed that, after delivery, reproductive hormones decrease rapidly, and this probably contributes to the onset of depression in susceptible mothers. Most likely, hormonal changes play a role in different factors leading to postpartum depression.

Other pregnancy-related conditions, such as preterm birth (before 34 weeks of gestation), or having a baby with a congenital defect, may also cause depression. This disorder also occurs when the current pregnancy is related to insecurities, like an unplanned pregnancy or having considered an abortion.

Some mothers do not have depression but describe a feeling of sadness or melancholy in the first three to five days after childbirth. When this occurs, nurses, obstetricians, and neonatologists may tell these mothers that they should not worry so much because such feelings will usually go away in the next two weeks. Still, 
it is absolutely necessary to make an appointment with them after discharge and, if possible, every week until the first month.

Therefore, it would be adequate to teach every mother to recognize postpartum depression symptoms and query them for the presence of symptoms that may be caused by depression, before and after delivery. With these actions, the best results possible will be most likely obtained through an early identification and management of postpartum depression in the first weeks.

Based on the disorders described here, in order to detect depression, it is necessary to perform a systematic screening of symptoms during pregnancy and especially during the first weeks after childbirth, in particular in women with risk factors. Screening is usually based on the Edinburgh Postnatal Depression Scale (EPDS). There is a Spanish version available that includes the 10 items and is very easy to use, so it may be administered in every hospital.

It has been used at Hospital Italiano de Buenos Aires for approximately 10 years. Pediatricians who administer the EPDS are able to detect the disorders leading to postpartum depression in the first weeks and, as soon as they do, an appropriate treatment is started.
Undoubtedly, it is necessary for Argentine hospitals to administer the EPDS in a systematic manner for the early detection of depression.

José María Ceriani Cernadas

Editor

http:/ / dx.doi.org/10.5546/aap.2020.eng.154

To cite: Ceriani Cernadas JM. Postpartum depression: Risks and early detection. Arch Argent Pediatr 2020;118(3):154-155.

\section{REFERENCES}

- Patel M, Bayley RK, Jabeen S, Ali S, Barker NC, Osiezagha K. Postpartum depression: a review. J Health Care Poor Underserved. 2012;23(2):534-42

- Mendoza C, Saldivia S. Actualización en depresión postparto: el desafío permanente de optimizar su detección y abordaje. Rev Med Chile 2015; 143: 887-894.

- Stewart DE, Vigod S. Postpartum depression. N Engl J Med 2016; 375: 2177-21.

- Moldenhauer JS. MSD Manual Professional Version. Postpartum depression. Last full review / revision Jun 2018. [Cited:27-03-2020]. Availableat:https: / / www.msdmanuals. $\mathrm{com} /$ professional / gynecology-and-obstetrics / postpartumcare-and-associated-disorders/ postpartum-depression. 